\title{
Modeling of Solar Wind in the Coronal Funnel with Mass and Energy Supplied at $5 \mathrm{Mm}$
}

\author{
J.-S. He • C.-Y. Tu • E. Marsch
}

Received: 18 December 2007 / Accepted: 26 May 2008 / Published online: 22 June 2008

(C) Springer Science+Business Media B.V. 2008

\begin{abstract}
The origin of the solar wind is a long-standing issue in both observational and theoretical studies. To understand how and where in the solar atmosphere the mass and energy of the solar wind are supplied is very important. Previous observation suggests a scenario in which the fast solar wind originates at heights above $5 \mathrm{Mm}$ in the magnetically open funnel, a process that is accompanied by downward flow below $5 \mathrm{Mm}$, whereby the mass and energy are supplied through reconnection between the open funnel and adjacent closed loops. Based on this scenario, we develop a fluid model to study the solar wind generation under the assumption that mass and energy are deposited in the open funnel at $5 \mathrm{Mm}$. The mass supply rate is estimated from the mass loss rate as given by the emptying of the side loops as a result of their assumed reconnection with the open funnel. Similarly, the energy input rate is consistent with the energy release rate as estimated from the energy flux associated with the reconnection between the open magnetic funnel and the closed magnetic loops. Following the observations, we not only simulate the plasma flowing upward to form the solar wind but also calculate the downward flow back to the lower atmosphere. This model is a first attempt to study physically the proposed scenario of solar wind origin and gives a new physical illustration of the possible initial deposition and consequent transportation of mass and energy in the coronal funnel.
\end{abstract}

J.-S. He · C.-Y. Tu (凶)

School of Earth and Space Sciences, Peking University, 100871 Beijing, China

e-mail: chuanyitu@pku.edu.cn

J.-S. He

e-mail: jshept@pku.edu.cn

C.-Y. Tu · E. Marsch

Max-Planck-Institut für Sonnensystemforschung, 37191 Katlenburg-Lindau, Germany

E. Marsch

e-mail: marsch@mps.mpg.de 


\section{Introduction}

The solar wind formation process is complex and remains under intensive investigation. It remains controversial in theoretical modeling as to from which layer of the solar atmosphere the solar wind actually starts flowing outward. The solar wind was originally thought to be generated at the coronal base (e.g., Parker, 1958; Whang and Chang, 1965), where the temperature was assumed to be as high as $10^{6} \mathrm{~K}$, so that no coronal heating needed to be taken into account. Afterward, in several studies of solar wind formation it was suggested that coronal heating was combined with solar wind acceleration (e.g., Hollweg, 1986). Various models were developed to investigate the solar wind origin by setting different lower boundary conditions: below the coronal base, for example, at the transition region (Tu and Marsch, 1997; Marsch and Tu, 1997; Hackenberg, Marsch, and Mann, 2000), or chromosphere (Hansteen and Leer, 1995; McKenzie, Sukhorukova, and Axford, 1998), as well as photosphere (Suzuki and Inutsuka, 2005). However, in all these previous models it was assumed that the fast solar wind is created on a single open field line without mass or energy being supplied from the sides but merely from below, and only upward flow was considered.

Recent observational diagnostics of the solar wind source regions greatly helped to derive a more realistic and complex picture of the solar wind origin. The solar wind was, by means of radiance and Doppler-blueshift maps, found to originate at the base of the chromospheric network structures in the polar coronal hole and in the high-latitude quiet-Sun region (Hassler et al., 1999; Wilhelm et al., 2000; Xia, Marsch, and Curdt, 2003; He, Tu, and Marsch, 2007). Ubiquitous downward flows existing in the lower transition region were inferred from Doppler redshifts of many ultraviolet emission lines, which are cooler than those appearing as Doppler blueshifts (e.g., Peter and Judge, 1999; Dammasch et al., 1999; Tu et al., 2005a, 2005b). The emission heights of lines stemming from various kinds of heavy tracer ions with different temperatures and velocities could be determined through correlation analysis between the radiance/Doppler-shift maps and the extrapolated magnetic structures (Marsch, Wiegelmann, and Xia, 2004; Tu et al., 2005a; Marsch et al., 2006). Furthermore, Tu et al. (2005a) proposed a concrete scenario of solar wind origin, assuming that small-scale closed loops are advected by the supergranular magneto-convection to reconnect with the open funnel, and thereby release their confined mass and stored magnetic energy to the open funnel. Thus they invoked reconnection to drive plasma flowing upward and to form solar wind above $5 \mathrm{Mm}$, as well as to push plasma moving downward below $5 \mathrm{Mm}$.

Following this scenario suggested by Tu et al. (2005a, 2005c), we here bring forward a new model to explain the solar wind origin. In this model it is assumed that mass and energy are deposited into the coronal funnel at $5 \mathrm{Mm}$ through some underlying magnetic interactions between the open funnel and surrounding loops, and thus the model combines upward and downward flows in the funnel together to describe the whole process of solar wind origin. In Section 2, we will first present the observational evidence and lay the foundation for modeling, and then describe in details the model for the upward-flow region as well as for downward-flow region. In Section 3, the numerical results of the model are illustrated and compared with observations. In Section 4, the mass-deposition rate used in the model is justified by comparing it with the estimated mass-release rate from the vanishing side loops, and the energy-deposition rate in the model is checked by comparison with the estimated energy-release rate from the assumed reconnection between open magnetic flux and closed flux in loops. A summary and discussion are presented in Section 5. 


\section{Model Description}

\subsection{Observational Basis for Modeling}

Tu et al. (2005a) studied the characteristics of various ion emission lines throughout the transition region in the polar coronal hole and found that Si II with field-averaged Doppler redshift emits at a lower transition region height, $\mathrm{C}$ IV with almost zero field-averaged Doppler shift emits at an intermediate transition region height, and Ne VIII with fieldaveraged Doppler blueshift emits at an upper transition region height. The results of Tu et al. (2005a) are listed in Table 1.

Stimulated by these observations, Tu et al. (2005a, 2005c) suggested a new way to explain how the fast solar wind may originate. The corresponding physical scenario is sketched in Figure 1(a), which illustrates that advection by supergranulation brings the closed loops together and forces them to collide and reconnect with the open funnel, a process by which mass and energy are delivered to the open funnel at $5 \mathrm{Mm}$ to support the upflow above $5 \mathrm{Mm}$ and the downflow below $5 \mathrm{Mm}$. A sketch of the resulting one-dimensional (1-D) modeling of such a scenario is illustrated in Figure 1(b). Plasma flows upward in Region 2 with its lower boundary at $5 \mathrm{Mm}$ and its upper boundary at the sonic point, the height of which is undetermined beforehand. Plasma flows downward in Region 1 with its upper boundary at $5 \mathrm{Mm}$ and its lower boundary at a height corresponding to a temperature of $T=2 \times 10^{4} \mathrm{~K}$, where hydrogen atoms begin to become fully ionized.

Table 1 Characteristics of various ion emission lines throughout the transition region in a polar coronal hole from data obtained by Tu et al. (2005a), which are the observational basis of our modeling.

\begin{tabular}{llcc}
\hline Ion emission line & Formation temperature $(\mathrm{K})$ & Doppler velocity $\left(\mathrm{km} \mathrm{s}^{-1}\right)$ & Correlation height $(\mathrm{Mm})$ \\
\hline Si II & $2.0 \times 10^{4}$ & $-2.0 \pm 1.3$ & 4 \\
C IV & $1.0 \times 10^{5}$ & $0.0 \pm 3.3$ & 5 \\
Ne VIII & $6.3 \times 10^{5}$ & $10.0 \pm 2.0$ & 24 \\
\hline
\end{tabular}
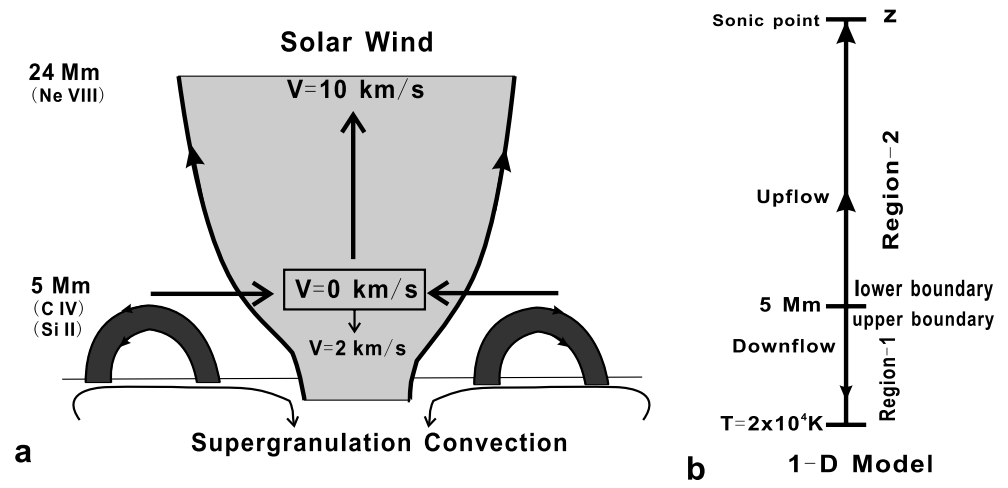

Figure 1 (a) Sketch to illustrate the scenario of the solar wind origin and mass supply and to show that supergranular convection can be the driver of solar wind outflow in coronal funnels (after Tu et al., 2005c). (b) Sketch of Region 1 and Region 2, the two computational domains in our model. Region 2 ranges from its lower boundary at $5 \mathrm{Mm}$ to its upper unfixed boundary at the sonic point. Region 1 ranges from its upper boundary at $5 \mathrm{Mm}$ to its lower boundary with a temperature of $2 \times 10^{4} \mathrm{~K}$, where hydrogen atoms begin to become fully ionized. Mass is deposited at $5 \mathrm{Mm}$ and flows upward in Region 2 and downward in Region 1. 


\subsection{Magnetic Flux Tube Configuration}

Following the work of Tu and Marsch (1997), Marsch and Tu (1997), and Hackenberg, Marsch, and Mann (2000) to describe flows in a open funnel in coronal hole, we design a magnetic flux tube in which solar plasma can flow upward and downward, with the variation of $B_{z}$, the radial component of magnetic field averaged over the cross section of the flux tube, shown as

$$
B_{z}(z)=B_{\text {funnel }}(z)+B_{\text {global }}(z),
$$

where $B_{\text {funnel }}(z)$ is the funnel magnetic field local component dominating the field variation over the lower region that ranges from the photosphere to a height of some tens of megameters and $B_{\text {global }}(z)$ is the global component dominating over the upper region in the solar atmosphere above some tens of megameters.

The global component $B_{\text {global }}(z)$ can be described with a function similar to Equation (2) in Banaszkiewicz, Axford, and McKenzie (1998), which reads

$$
B_{\text {global }}(r)=M\left[\frac{2}{r^{3}}+\frac{3 Q}{r^{5}}+\frac{K}{a_{1}\left(r+a_{1}\right)^{2}}\right],
$$

where $r$ is the normalized distance, $r=z / R_{\odot}+1$, and the parameters of Equation (2) are set as $M=1.789 \mathrm{G}, Q=0.26, K=1.0$, and $a_{1}=1.538$. This model expression describes the radial component of magnetic field along the straight polar field line, which is composed of three components: The first term on the right-hand side (RHS) represents the dipolar contribution, the second one a quadrupolar contribution, and the third one the current-sheet contribution to the total field.

The funnel component $B_{\text {funnel }}(z)$, which strongly decreases with height in the lower region and finally approaches zero in the upper region, can be assumed to vary according to an exponential function as

$$
B_{\text {funnel }}(z)=b_{0} \cdot \exp \left(b_{1} \cdot z\right),
$$

where the parameters $b_{0}$ and $b_{1}$ are $56.5 \mathrm{G}$ and $-0.28 \mathrm{Mm}^{-1}$, respectively, which are obtained by using Equation (3) to fit the residual funnel component values, which are obtained by subtracting the calculated global component values from the extrapolated values $(\mathrm{Tu}$ et al., 2005c).

The shape of the magnetic flux tube according to Equation (1) is illustrated in Figure 2, which expands rapidly in the lower region $(<20 \mathrm{Mm})$ and expands slowly in the upper region $(>20 \mathrm{Mm}$ ). The source region of solar wind is set at a height of $5 \mathrm{Mm}$ in the flux tube, below which plasma flows downward, resulting in redshift of emission lines, and above which plasma flows upward, resulting in blue-shift of emission lines (Tu et al., 2005a). The location of mass and energy supply originating from the assumed reconnections is placed at $5 \mathrm{Mm}$. The size of the flux tube is set consistent with the one shown in Tu et al. (2005a). The cross-sectional area of the flux tube at a relative height of $0 \mathrm{Mm},\left.A\right|_{z=0 \mathrm{Mm}}$, is $53 \mathrm{Mm}^{2}$, and thus according to magnetic flux conservation, the cross-sectional area at $5 \mathrm{Mm},\left.A\right|_{z=5 \mathrm{Mm}}$, is $188 \mathrm{Mm}^{2}$ in Figure 2.

\subsection{Description of the Governing Fluid Equations}

The mass, momentum, and energy equations governing the steady flows in Region 1 and Region 2 are

$$
\frac{\partial}{\partial z}(\rho v A)=0
$$


Figure 2 A side view of the magnetic flux tube shape, which is cylindrically symmetric around the radial axis $x=0 \mathrm{Mm}$. The expansion of the flux tube is determined by the radial component $B_{z}$ averaged over the cross section, which varies along with the height $z$ according to Equation (1). The flux tube is divided into two regions, Region 1 ( $<5 \mathrm{Mm})$ and Region 2 ( $>5 \mathrm{Mm}$ ), by the bold dashed dividing line at $5 \mathrm{Mm}$. Plasma flows downward in Region 1 and upward in Region 2 and their flow directions are marked by a downward arrow and an upward one, respectively.

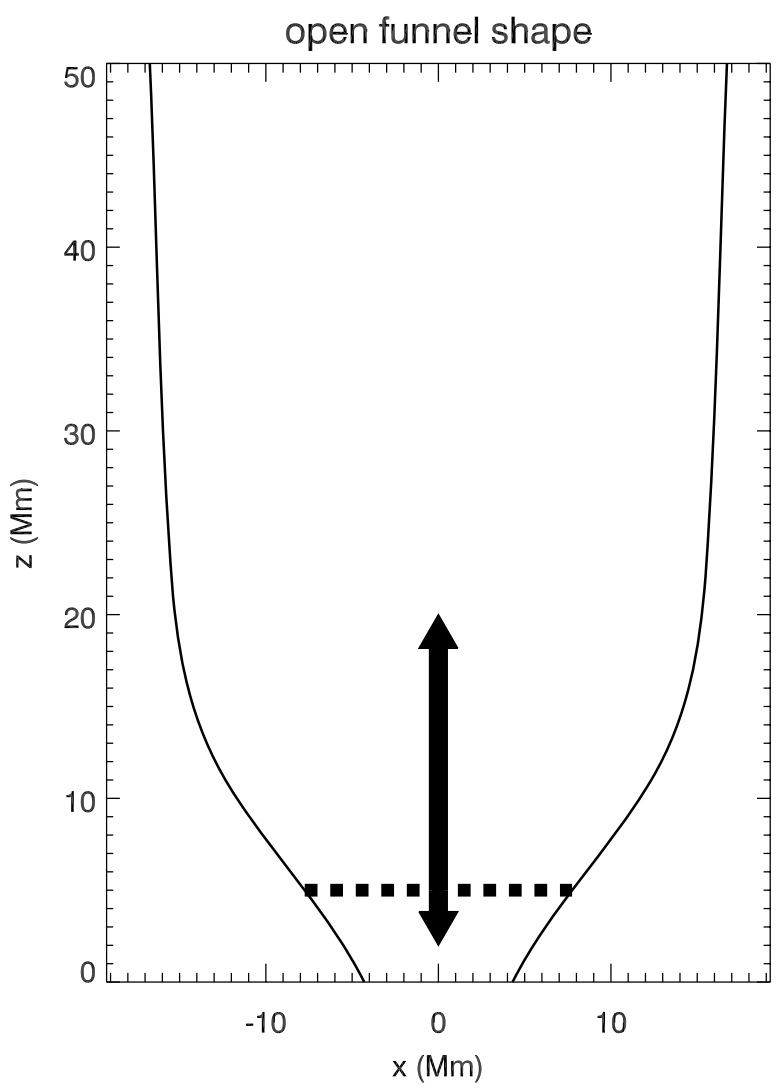

$$
\begin{gathered}
\rho v \frac{\partial}{\partial z} v=-\frac{\partial}{\partial z}\left(P+P_{\mathrm{A}}\right)-\rho g, \\
\frac{v \cdot 2 n k_{\mathrm{B}}}{\gamma-1} \frac{\partial}{\partial z} T+\frac{2 n k_{\mathrm{B}} T}{A} \frac{\partial}{\partial z}(A v)=-L_{r}+H-\frac{1}{A} \frac{\partial q}{\partial z},
\end{gathered}
$$

respectively, where the expressions of some terms for Region 1 are different from those for Region 2 . Here $\rho$ is the mass density, $v$ is the flow velocity, $A$ is the cross-sectional area of the flux tube, $T$ is the temperature, and $q$ is the thermal conduction flux. The thermal pressure $P$ in Equation (5) is set to $2 n k_{\mathrm{B}} T$, with $n$ being the number density. The Alfvénic wave pressure $P_{\mathrm{A}}$ in Equation (5) is expressed as

$$
P_{\mathrm{A}}=\frac{\left\langle\delta B^{2}\right\rangle}{8 \pi}=\frac{1}{8 \pi} \int_{f_{\mathrm{L}}}^{f_{\mathrm{H}}} P(f, \mathrm{z}) \mathrm{d} f,
$$

where $P(f, z)$ represents the power spectrum density of frequency $f$ at a height of $z$. The frequency $f_{\mathrm{H}}$ denotes the upper threshold, above which the wave is damped through resonance with ambient plasma ions, and the frequency $f_{\mathrm{L}}$ is a lower threshold, below which the wavelength $\lambda\left(=V_{\mathrm{A}} / f\right)$ is too long to satisfy the linear WKB approximation for wave propagation. The upper threshold $f_{\mathrm{H}}$ is taken as $\alpha_{f} \frac{1}{2 \pi} \frac{e B}{m_{p} c}$ in Region 2, with $\alpha_{f}$ being 0.1 , which is the same value as that in Tu and Marsch (1997). The value of $f_{\mathrm{H}}$ stays constant 
in Region 1 owing to nonresonance of waves with ions in this region. The lower threshold $f_{\mathrm{L}}$ is $1.0 \mathrm{~Hz}$ for Region 1 and Region 2 . The spatial transport process of the wave spectrum $P(f, z)$, which is assumed to have a slope of -1 in the whole frequency domain, can be described by the WKB approximation and is thus expressed as

$$
P(f, z)=P\left(f_{\mathrm{L}}, z_{0}\right) \cdot \frac{f_{\mathrm{L}}}{f} \cdot \frac{M_{\mathrm{A}}\left(z_{0}\right) \cdot\left[1+M_{\mathrm{A}}\left(z_{0}\right)\right]^{2}}{M_{\mathrm{A}}(z) \cdot\left[1+M_{\mathrm{A}}(z)\right]^{2}},
$$

which depends on the Alfvén Mach number $M_{\mathrm{A}}=v / V_{\mathrm{A}}$.

The radiation loss $L_{r}$ in Equation (6) can be described as a piecewise function of the temperature according to Rosner, Tucker, and Vaiana (1978). The heating function $H$ in Equation (6) is tailored after Tu and Marsch (1997) and reads as follows:

$$
H(z)=-\left(v+V_{\mathrm{A}}\right) \frac{P\left(f_{\mathrm{H}}, z\right)}{4 \pi} \frac{\partial}{\partial z} f_{\mathrm{H}}(z) .
$$

The thermal conduction flux $q$ in Equation (6) is expressed as

$$
q=-\kappa(T) \cdot A \frac{\partial T}{\partial z}
$$

where the thermal conduction coefficient $\kappa(T)$ is defined as

$$
\kappa=\left(1+\frac{1}{24.8}\right) \cdot \kappa_{0} \cdot T^{5 / 2}
$$

and where $\kappa_{0}$ is determined by the Coulomb logarithm $\ln \Lambda$ with the following expression given by Hinton (1983):

$$
\kappa_{0}=1.84 \times 10^{-6} \cdot\left(\frac{\ln \Lambda}{10}\right)^{-1} .
$$

The Coulomb logarithm $\ln \Lambda$ is set to 13 in Region 1 for $n=0.8 \times 10^{9} \mathrm{~cm}^{-3}$ and $T=2.5 \times 10^{4} \mathrm{~K}$ and is set to 18 in Region 2 for $n=1.0 \times 10^{8} \mathrm{~cm}^{-3}$ and $T=$ $3.0 \times 10^{5} \mathrm{~K}$. The resulting $\kappa_{0}$ values are $1.39 \times 10^{-6} \mathrm{erg} \mathrm{cm}^{-1} \mathrm{~s}^{-1} \mathrm{~K}^{-7 / 2}$ in Region 1 and $1.02 \times 10^{-6} \mathrm{erg} \mathrm{cm}^{-1} \mathrm{~s}^{-1} \mathrm{~K}^{-7 / 2}$ in Region 2 .

Following Hackenberg, Marsch, and Mann (2000), we can transform the governing partial differential Equations (4), (5), and (6), together with the thermal conduction flux definition (Equation (10)), into a closed set of governing ordinary differential equations:

$$
\begin{gathered}
\frac{\mathrm{d} \rho}{\mathrm{d} z}=\frac{\rho}{A} \frac{\dot{M}^{2} A^{\prime}+A^{2} \rho^{2} \frac{2 k_{\mathrm{B}}}{m}(q / \kappa)-A^{3} \rho^{2} \tilde{g}}{A^{2} \rho P_{\mathrm{eff}}-\dot{M}^{2}}, \\
\frac{\mathrm{d} T}{\mathrm{~d} z}=-\frac{q}{\kappa A}, \\
\frac{\mathrm{d} q}{\mathrm{~d} z}=A\left(H-L_{r}\right)+\frac{\dot{M} k_{\mathrm{B}}}{m}\left(\frac{2 T \rho^{\prime}}{\rho}-\frac{2 T^{\prime}}{\gamma-1}\right),
\end{gathered}
$$


where $A^{\prime}, \rho^{\prime}$, and $T^{\prime}$ are $\frac{\mathrm{d} A}{\mathrm{~d} z}, \frac{\mathrm{d} \rho}{\mathrm{d} z}$, and $\frac{\mathrm{d} T}{\mathrm{~d} z}$, respectively, $\dot{M}$ in Equation (13) is the mass flux, $P_{\text {eff }}$ in Equation (13) is the total effective pressure defined as

$$
P_{\text {eff }}=2 n k_{\mathrm{B}} T+\frac{1+4 M_{\mathrm{A}}+3 M_{\mathrm{A}}^{2}}{2+4 M_{\mathrm{A}}+2 M_{\mathrm{A}}^{2}} P_{\mathrm{A}},
$$

and $\tilde{g}$ in Equation (13) is a modified effective gravitational acceleration defined as

$$
\tilde{g}=g+\frac{P_{\mathrm{A}}}{\rho \omega_{\mathrm{H}} \ln \frac{\omega_{\mathrm{H}}}{\omega_{\mathrm{L}}}} \frac{\partial}{\partial z} \omega_{\mathrm{H}} .
$$

The interface between Region 1 and Region 2 is assumed to be located at $5 \mathrm{Mm}$. The mass densities, velocities, temperatures, and wave pressures at the upper boundary in Region 1 and the lower boundary in Region 2 must be assigned carefully to conform to momentum governing Equation (5) in the vicinity of the interface. The integral form of Equation (5) across the interface reads as

$$
\left.\rho v^{2}\right|_{z^{*}-\Delta z} ^{z^{*}+\Delta z}+\left.P\right|_{z^{*}-\Delta z} ^{z^{*}+\Delta z}+\left.P_{\mathrm{A}}\right|_{z^{*}-\Delta z} ^{z^{*}+\Delta z}=0
$$

where $z^{*}(=5 \mathrm{Mm})$ is the interface height, $\Delta z$ is an infinitely small height interval, $z^{*}-\Delta z$ is the height in Region 1 near its upper boundary, and $z^{*}+\Delta z$ is the height in Region 2 near its lower boundary. A possible solution for Equation (18) is obtained with $\left.\rho\right|_{z^{*}-\Delta z}=\left.\rho\right|_{z^{*}+\Delta z}$, $\left.v\right|_{z^{*}-\Delta z}=-\left.v\right|_{z^{*}+\Delta z},\left.P\right|_{z^{*}-\Delta z}=\left.P\right|_{z^{*}+\Delta z}$, and $\left.P_{\mathrm{A}}\right|_{z^{*}-\Delta z}=\left.P_{\mathrm{A}}\right|_{z^{*}+\Delta z}$.

The mass density $\rho$ and velocity $v$ at the lower boundary in Region 2 are set as $8.65 \times$ $10^{-16} \mathrm{~g} \mathrm{~cm}^{-3}$ and $+3.3 \mathrm{~km} \mathrm{~s}^{-1}$, respectively, producing a mass flux density equivalent to the mass flux density measured at $1 \mathrm{AU}$ with a number density of $3 \mathrm{~cm}^{-3}$ and a velocity of $750 \mathrm{~km} \mathrm{~s}^{-1}$. The mass density $\rho$ at the upper boundary in Region 1 is also set as $8.65 \times$ $10^{-16} \mathrm{~g} \mathrm{~cm}^{-3}$, and the velocity there is set as $-3.3 \mathrm{~km} \mathrm{~s}^{-1}$. Thus the unsigned mass flux flowing both upward and downward in the open funnel $\Phi_{\mathrm{m}}\left(=2 \dot{M}=\left.2 \rho v A\right|_{\text {funnel, } 5 \mathrm{Mm}}\right)$ is $1.08 \times 10^{9} \mathrm{~g} \mathrm{~s}^{-1}$. This mass flux must be consistent with the mass input rate estimated from the assumed reconnections between central open funnel and side closed loops, which is in detail discussed in Section 4. The absolute value of the velocity at the boundary is taken as the standard deviation of the (on average zero) Doppler velocity of $\mathrm{C}^{3+}$ in the coronal hole region (Tu et al., 2005a), thus taking the variance of the carbon ion speed as tracer for the initial proton speed.

The temperatures $T$ at the lower boundary in Region 2 and at the upper boundary in Region 1 are both assigned the value $10^{5} \mathrm{~K}$, which is the formation temperature of the $\mathrm{C}$ IV line used here (Tu et al., 2005a).

The wave pressure $P_{\mathrm{A}}$ at the lower boundary in Region 2 is derived from the wave energy flux density $F_{\mathrm{A}}$, by using the relation $P_{\mathrm{A}}=F_{\mathrm{A}} /\left(3 v+2 V_{\mathrm{A}}\right)$ of the WKB approximation. The input value of the wave energy flux density $F_{\mathrm{A}}$ at the lower boundary in Region 2 can be roughly estimated from the sum of the increment of solar wind kinetic energy and the drop of gravitational potential energy from $5 \mathrm{Mm}$ to $1 \mathrm{AU}$. Therefore, the required wave pressure $P_{\mathrm{A}}$ is roughly estimated to be $0.31 \times 10^{-2} \mathrm{dyn} \mathrm{cm}^{-2}$, which may be a little too small as a result of neglecting the radiation loss in this rough estimation. Two possible values of $P_{\mathrm{A}}$ at the lower boundary in Region 2 (i.e., $0.45 \times 10^{-2}$ and $0.52 \times 10^{-2} \mathrm{dyn} \mathrm{cm}^{-2}$ ) are taken as examples for the modeling. The wave pressure $P_{\mathrm{A}}$ at the upper boundary in Region 1 is chosen to be the same as that at the lower boundary in Region 2. The wave phase speed $V_{\mathrm{A}}$ at the upper boundary in Region 1 is opposite to that at the lower boundary in Region 2 . The summed wave energy flux and kinetic energy flux in both upward and downward directions falls in 
the range of the energy release rate as produced by the assumed reconnection between the open funnel and its adjacent loops, as is discussed in detail in Section 4.

The thermal conduction flux $q$ at the lower boundary in Region 2 is left as a parameter to be adjusted during the numerical computation for the purpose of going with the solution through Parker's sonic critical point, at which both numerator and denominator on the RHS of Equation (13) must become zero simultaneously. The thermal conduction flux $q$ at the upper boundary in Region 1 is adjusted to get a reasonable solution with temperature decreasing to $2 \times 10^{4} \mathrm{~K}$ at the smallest value of $z$.

A fifth-order-accurate Runge - Kutta integration scheme with adaptive step-size control (Press et al., 1992) is used to integrate the governing Equations (13), (14), and (15). In Region 2, the numerical integration is processed with $q$ at the lower boundary being determined by the shooting method (Press et al., 1992) to assure a simultaneous approach to zero for numerator and denominator on the RHS of Equation (13). In Region 1, $q$ is obtained by adjusting it to satisfy that plasma flows downward to the Region 1 lower boundary with temperature decreasing to $2 \times 10^{4} \mathrm{~K}$.

\section{Model Results}

The variations of $\rho, v, T$, and $q$ along with the height in the funnel for Region 1 and Region 2 are calculated and shown in Figures 3 and 4. The downward flow in Region 1 and the upward flow in the beginning segment of Region 2 are shown in Figure 3. The plasma moves upward with increasing velocity and increasing temperature in the beginning section of Region 2. The velocity profile against the height in Figure 3(b) shows a curvature in the height range from 5 to $10 \mathrm{Mm}$ as a result of the strong acceleration of the plasma near $5 \mathrm{Mm}$ by the large gradient force of the wave pressure. In Region 1, the plasma moving downward slows and becomes cooler, which may be related with the observation that ion emission lines tend to become less redshifted when their formation temperature decreases (Peter and Judge, 1999). The plasma flowing upward is heated from $1 \times 10^{5} \mathrm{~K}$ at $5 \mathrm{Mm}$ to $7 \times 10^{5} \mathrm{~K}$ at $24 \mathrm{Mm}$, approximately consistent with the observations that the $\mathrm{C}$ IV line with a formation temperature of $1 \times 10^{5} \mathrm{~K}$ is emitted at $5 \mathrm{Mm}$, and the Ne VIII line with a formation temperature of $6.3 \times 10^{5} \mathrm{~K}$ is emitted at $24 \mathrm{Mm}$ (Tu et al., 2005a). The plasma flowing downward is cooled from 5 to $4.45 \mathrm{Mm}$, approximately consistent with the observation that Si II with a formation temperature of $2 \times 10^{4} \mathrm{~K}$ is emitted at $4 \pm 3 \mathrm{Mm}$ (Tu et al., 2005a). For two values of $P_{\mathrm{A}}$, we have made the corresponding numerical computations, the results of which are shown by the solid and dotted lines in Figure 3, respectively.

Figure 4 illustrates the upward flow throughout Region 2. Plasma flowing upward is further accelerated to the sound speed reached at Parker's critical sonic point (Figure 4(b)). Meanwhile, the calculated number density of the solar wind decreases, and this trend is similar to that inferred from SUMER observations (Wilhelm et al., 1998) (Figure 4(a)). The temperature increases rapidly to $8 \times 10^{5} \mathrm{~K}$ near $30 \mathrm{Mm}$, and then further increases slowly with a trend approximating the temperatures derived from SUMER observations (Wilhelm et al., 1998) (Figure 4(c)).

\section{Discussion of the Mass and Energy Input of the Model}

Our model vitally relies on the mass and energy deposited into the open funnel at $5 \mathrm{Mm}$, both of which are supposed to be delivered through reconnection between the open funnel 

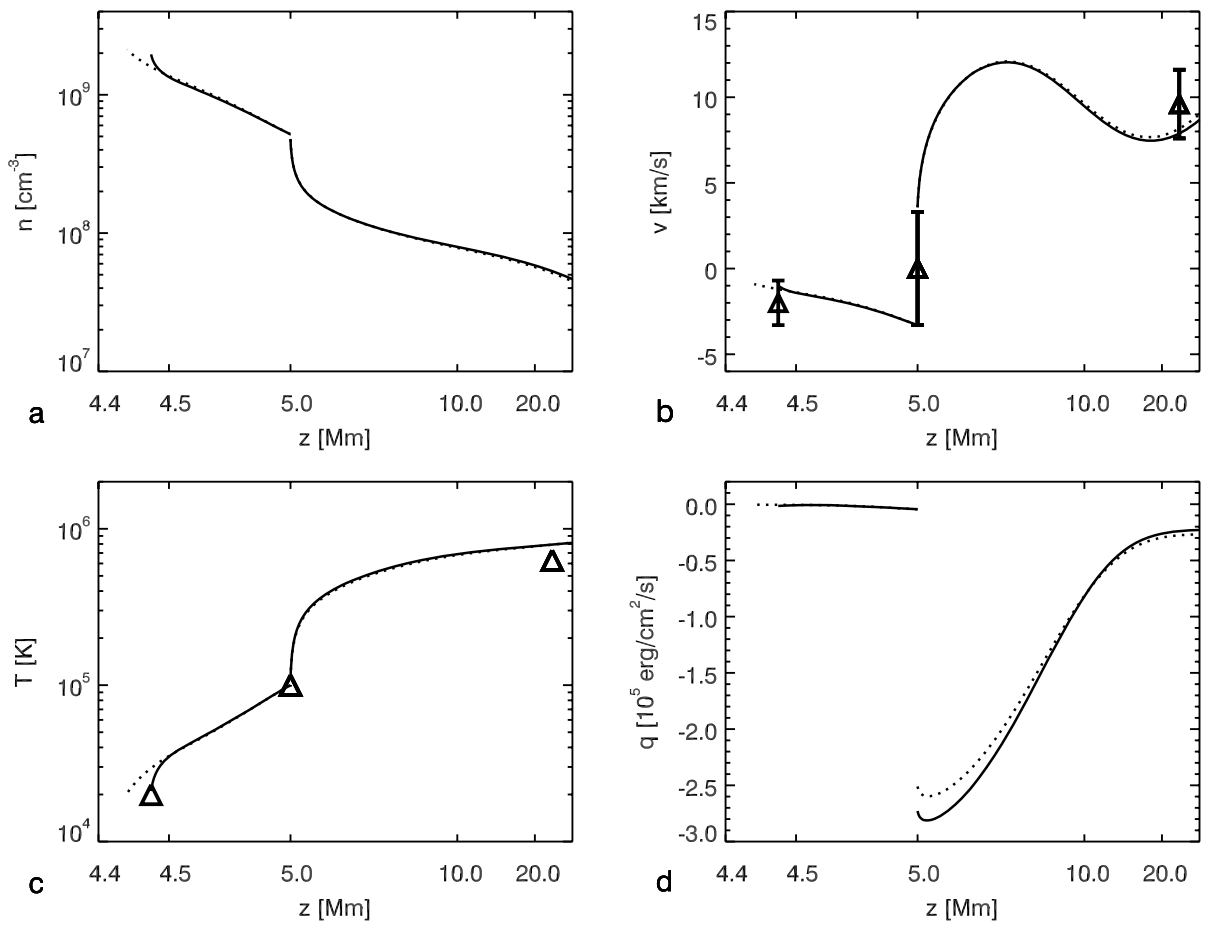

Figure 3 Variation of coronal plasma parameters with height throughout Region 1 and in the beginning segment of Region 2. The solid lines in the four panels are for a wave pressure $P_{\mathrm{A}}$ of $0.52 \times 10^{-2} \mathrm{dyn} \mathrm{cm}^{-2}$ at $5 \mathrm{Mm}$, and the dotted lines for a pressure of $0.45 \times 10^{-2} \mathrm{dyn} \mathrm{cm}^{-2}$ at $5 \mathrm{Mm}$. The situation for the larger $P_{\mathrm{A}}$ is described as an example. (a) Profiles of number density as a function of height in Region 1 and in Region 2. (b) The left solid line is the velocity profile in Region 1; the right solid line is for the velocity profile in Region 2. The leftmost triangle is about the field-averaged Doppler velocity for the observed Si II red shift, and the error bar corresponds to the standard deviation of the measured Doppler velocity. The middle triangle and error bar on it represent, respectively, the mean and standard deviation of the measured C IV Doppler velocity. The Doppler velocity of Ne VIII is shown as the rightmost triangle and error bar. (c) The solid line on the left of $5 \mathrm{Mm}$ gives the temperature variation of plasma flowing downward in Region 1 . The solid line beyond $5 \mathrm{Mm}$ shows the temperature profile of plasma moving upward in Region 2. The leftmost, middle, and rightmost triangles denote the formation temperatures of Si II, C IV, and Ne VIII, respectively. (d) The solid line on the left is the height profile of the thermal conduction flux in Region 1, and the one on the right is for the thermal conduction flux in Region 2.

and side closed loops as implied by the observations (Tu et al., 2005a, 2005c). In this section, we present a quantitative estimation of the mass and energy input rate from assumed reconnection, which is required for our modeling. We assume that the mass of the closed loops is completely delivered into the open funnel through reconnection during which the loops vanish. The mass input rate is estimated with

$$
\Phi_{\mathrm{m}}^{*}=\frac{N_{\text {loop }} \cdot M_{\text {loop }}}{\Delta t},
$$

where $\Phi_{\mathrm{m}}^{*}$ is the mass input rate, $N_{\text {loop }}$ is the number of intranetwork (IN) closed loops that simultaneously exist at any time around an open funnel located at a network intersection, $\Delta t$ is the typical lifetime of any loop, and $M_{\text {loop }}$ is the mass stored in a closed loop before reconnection. The number of closed loops, $N_{\text {loop }}$, is set to 5 according to Zhang, Ma, and 

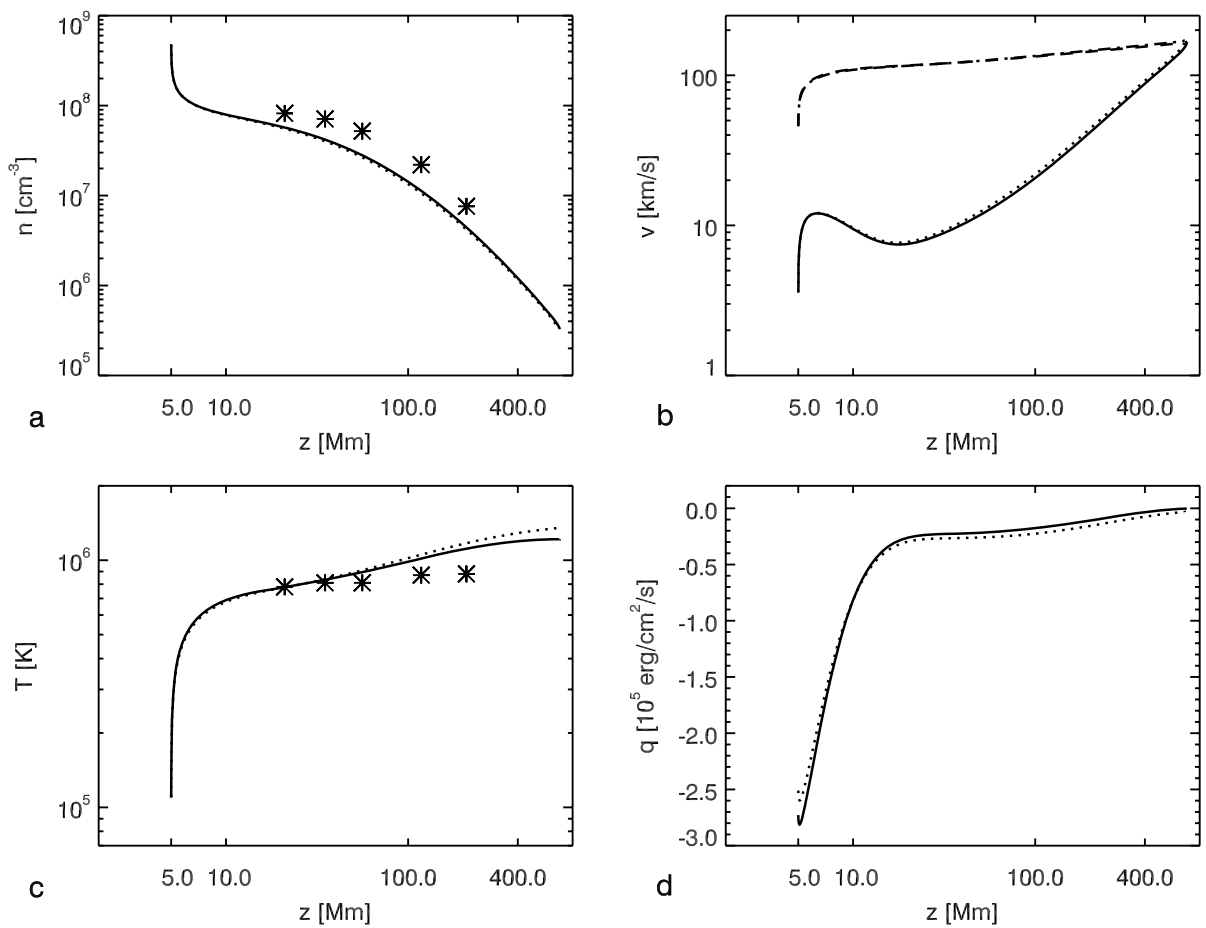

Figure 4 Variations of solar wind parameters with height throughout Region 2. The styles of lines, solid and dotted, have the same meaning as in Figure 3. As an example, we describe the situation for larger $P_{\mathrm{A}}$. (a) The solid line represents the number density variation above $5 \mathrm{Mm}$. The asterisks are the number densities as derived from polar interplume observations in Si VIII given by Wilhelm et al. (1998). (b) The solid line is the plasma velocity profile and the dashed line is for the effective sound speed $V_{\text {eff, }}\left(=\sqrt{P_{\text {eff }} / \rho}\right)$ in the flowing plasma. (c) The solid line shows the temperature variation with height above $5 \mathrm{Mm}$. The asterisks are the temperature values derived from polar interplume observations in Mg IX given by Wilhelm et al. (1998). (d) The solid line gives the height profile of the thermal conduction flux above $5 \mathrm{Mm}$.

Wang (2006). The lifetime of a closed loop, $\Delta t$, is estimated as 2.1 hours for an IN element with the most probable magnetic flux of $6.0 \times 10^{16} \mathrm{Mx}$ according to Zhang et al. (1998a, $1998 \mathrm{~b}$ ). These $N_{\text {loop }}$ closed loops are thought to be advected to the open funnel through supergranular convection and to deliver the confined mass to the open funnel through magnetic reconnection. The mass contained in a single closed loop, $M_{\text {loop }}$, is estimated on the basis of Equation (20), by assuming that the closed loop stays in hydrostatic equilibrium and has a semicircular shape, an isothermal temperature, and a uniform cross section. Then its mass is

$$
\begin{aligned}
M_{\text {loop }}= & 2 A_{\text {loop }} \cdot \int_{0}^{h_{\text {loop }}} \frac{P_{\text {loop, base }}}{T_{\text {loop }}} \cdot m \\
& \cdot \exp \left(-\frac{G M_{\odot} m}{2 R_{\odot} k_{\mathrm{B}} T_{\text {loop }}} \cdot z\right) \frac{h_{\text {loop }}}{\sqrt{h_{\text {loop }}^{2}-z^{2}}} \mathrm{~d} z .
\end{aligned}
$$

The uniform cross-sectional area $A_{\text {loop }}$ in Equation (20) is estimated as $3.15 \times 10^{16} \mathrm{~cm}^{2}$ $\left(=6.25 \operatorname{arcsec}^{2}\right)$ through an eye measurement of the IN elements in Figure 3 of Zhang et al. (1998b), since the exact mean area of the IN elements with the most probable magnetic 
flux is not given in Zhang et al. (1998b). The apex height $h_{\text {loop }}$, namely the radius of the semicircular shape, is assumed to be $5 \mathrm{Mm}$. The isothermal temperature $T_{\text {loop }}$ is set as $10^{5} \mathrm{~K}$. The electron pressure at the loop base, $P_{\text {loop,base }}$, is taken as $4 \times 10^{15} \mathrm{~K} \mathrm{~cm}^{-3}$ in the coronal hole cell center where closed loops reside (Del Zanna and Bromage, 1999). The mass $M_{\text {loop }}$ is then evaluated to be $1.13 \times 10^{12} \mathrm{~g}$. The resulting mass input rate $\Phi_{\mathrm{m}}^{*}$ from side closed loops to the open funnel is estimated to be $0.75 \times 10^{9} \mathrm{~g} \mathrm{~s}^{-1}$. The mass flux in both downward and upward directions in the present model, $\Phi_{\mathrm{m}}$, is $1.08 \times 10^{9} \mathrm{~g} \mathrm{~s}^{-1}$, which is on the order of $\Phi_{\mathrm{m}}^{*}$ but a little larger than $\Phi_{\mathrm{m}}^{*}$.

The energy input rate into the open funnel via reconnection is thought to be smaller than the upper limit $\Phi_{E, \max }\left(=\left.2 n v A \frac{m_{p} \cdot V_{\mathrm{A}}^{2}}{2}\right|_{z=5 \mathrm{Mm}}\right)$, which means that energy is supplied in proportion of the mass flux with a specific proton kinetic energy as determined by the local Alfvén speed. Here the mass flux at $5 \mathrm{Mm},\left.2 \mathrm{~nm}_{p} v A\right|_{z=5 \mathrm{Mm}}$, namely $\Phi_{\mathrm{m}}^{*}$, amounts to $0.75 \times 10^{9} \mathrm{~g} \mathrm{~s}^{-1}$ as already discussed. The Alfvén wave phase speed at $5 \mathrm{Mm}$ in the open funnel, $\left.V_{\mathrm{A}}\right|_{z=5 \mathrm{Mm}}$, is calculated to be $1726 \mathrm{~km} \mathrm{~s}^{-1}$, according to $V_{\mathrm{A}}=B_{z} / \sqrt{4 \pi \rho}$. Therefore, the upper limit of the energy input rate $\Phi_{E \text {, max }}$ is estimated to be $1.12 \times 10^{25} \mathrm{erg} \mathrm{s}^{-1}$. The energy deposited supports the initial kinetic energy flux as well as the wave energy flux at the boundary. The energy deposition rate at $5 \mathrm{Mm}$ used in our model can be expressed as $\left.\Phi_{E}\right|_{z=5 \mathrm{Mm}}=\Phi_{\mathrm{m}} \cdot \frac{1}{2} v^{2}+\left.2 \cdot A \cdot P_{\mathrm{A}} \cdot\left(3 v+2 V_{\mathrm{A}}\right)\right|_{z=5 \mathrm{Mm}}$, which is calculated to be $5.9 \times 10^{24} \mathrm{erg} \mathrm{s}^{-1}$ for $P_{\mathrm{A}}=0.45 \times 10^{-2} \mathrm{dyn} \mathrm{cm}^{-2}$ and $6.8 \times 10^{24} \mathrm{erg} \mathrm{s}^{-1}$ for $P_{\mathrm{A}}=0.52 \times 10^{-2} \mathrm{dyn}^{-2}$, respectively. These two energy deposition rate used in our model are both less than the upper limit.

\section{Summary and Discussion}

In this paper, we have presented a new model to simulate the upward plasma flow forming the solar wind as well as simultaneous downward flow. These two flows together result in sizable Doppler shifts, which can explain the blueshifts observed in hotter emission lines as well as the redshifts seen in cooler emission lines, respectively. The modeling results generally agree with the observational results from the analysis by Tu et al. (2005a) and Wilhelm et al. (1998). The mass and energy input rates used in our model are shown to be sufficient to power the solar wind and consistent with an estimation of the possible mass and energy supplies through the assumed reconnection between the fields of the converging loops and funnel. This numerical model supports the scenario of solar wind origin as proposed by $\mathrm{Tu}$ et al. (2005a), in which it is conjectured that reconnections between lateral closed loops and a central open funnel can give rise to solar wind outflow as well as return flow to the solar surface in a magnetic funnel.

In future work, this model needs to be extended to a fully three-dimensional simulation and one needs to introduce the necessary and appropriate boundary conditions at the photosphere, which through magneto-convection enforce magnetic reconnection between open funnels and closed loops self-consistently.

Our model just indicates one possible mechanism for the solar wind origin, namely that the solar wind may be supplied with mass and energy from underlying magnetic reconnection between open flux tubes and closed loops. There are other possible mechanisms for the solar wind origin; for example, the solar wind may be driven by dissipation of low-frequency Alfvén waves, which are excited by footpoint motions on the photosphere (Suzuki and Inutsuka, 2005; Suzuki and Inutsuka, 2006). The low-frequency Alfvén waves have been identified from SOT/Hinode observations of spicules and a prominence (De Pontieu et al., 2007; Okamoto et al., 2007). The high-frequency Alfvén waves have not yet been measured in the 
solar wind source region. They are supposed to be generated from magnetic reconnection in the network (Axford and McKenzie, 1992). The observational data with high spatial and temporal resolution from Hinode may provide a good opportunity to check the real effects of the low-frequency Alfvén waves on the solar wind origin.

Acknowledgements We would like to thank Prof. Jun Zhang for many useful discussions. This work is supported by the National Natural Science Foundation of China under Contract Nos. 40574078, 40336053, and 40436015, the Foundation of Major Projects of National Basic Research under Contract No. 2006CB806305, and the Beijing Education Project XK100010404.

\section{References}

Axford, W.I., McKenzie, J.F.: 1992, In: Marsch, E., Schwenn, R. (eds.) Solar Wind Seven, COSPAR Colloq. 3, 1 .

Banaszkiewicz, M., Axford, W.I., McKenzie, J.F.: 1998, Astron. Astrophys. 337, 940.

Dammasch, I.E., Wilhelm, K., Curdt, W., Hassler, D.M.: 1999, Astron. Astrophys. 346, 285.

De Pontieu, B., McIntosh, S.W., Carlsson, M., Hansteen, V.H., Tarbell, T.D., Schrijver, C.J., et al.: 2007, Science 318, 1574.

Del Zanna, G., Bromage, B.J.I.: 1999, In: Vial, J.-C., Kaldeich-Schümann, B. (eds.) Plasma Dynamics and Diagnostics in the Solar Transition Region and Corona, Proc. 8th SOHO Workshop SP-446, ESA, Noordwijk, 269.

Hackenberg, P., Marsch, E., Mann, G.: 2000, Astron. Astrophys. 360, 1139.

Hansteen, V.H., Leer, E.: 1995, J. Geophys. Res. 100, 21577.

Hassler, D.M., Dammasch, I.E., Lemaire, P., Brekke, P., Curdt, W., Mason, H.E., Vial, J.-C., Wilhelm, K.: 1999, Science 283, 810.

He, J.-S., Tu, C.-Y., Marsch, E.: 2007, Astron. Astrophys. 468, 307.

Hinton, F.L.: 1983, In: Galeev, A.A., Sudan, R.N. (eds.) Basic Plasma Physics I, Handbook of Plasma Physics 1, North-Holland, Amsterdam, 147.

Hollweg, J.V.: 1986, J. Geophys. Res. 91, 4111.

Marsch, E., Tu, C.-Y.: 1997, Solar Phys. 176, 87.

Marsch, E., Wiegelmann, T., Xia, L.-D.: 2004, Astron. Astropyhs. 428, 629.

Marsch, E., Zhou, G.-Q., He, J.-S., Tu, C.-Y.: 2006, Astron. Astropyhs. 457, 699.

McKenzie, J.F., Sukhorukova, G.V., Axford, W.I.: 1998, Astron. Astrophys. 330, 1145.

Okamoto, T.J., Tsuneta, S., Berger, T.E., Ichimoto, K., Katsukawa, Y., Lites, B.W., et al.: 2007, Science 318, 1577.

Parker, E.N.: 1958, Astrophys. J. 128, 664.

Peter, H., Judge, P.G.: 1999, Astrophys. J. 552, 1148.

Press, W.H., Teukolsky, S.A., Vetterling, W.T., Flannnery, B.P.: 1992, Numerical Recipes in Fortran: The Art of Scientific Computing, Cambridge University Press, Cambridge.

Rosner, R., Tucker, W.H., Vaiana, G.S.: 1978, Astrophys. J. 220, 643.

Suzuki, T.K., Inutsuka, S.-I.: 2005, Astrophys. J. 632, L49.

Suzuki, T.K., Inutsuka, S.-I.: 2006, J. Geophys. Res. 111, A06101.

Tu, C.-Y., Marsch, E.: 1997, Solar Phys. 171, 363.

Tu, C.-Y., Zhou, C., Marsch, E., Xia, L.-D., Zhao, L., Wang, J.-X., Wilhelm, K.: 2005a, Science 308, 519.

Tu, C.-Y., Zhou, C., Marsch, E., Wilhelm, K., Xia, L.-D., Zhao, L., Wang, J.-X.: 2005b, Astrophys. J. Lett. 624, L133.

Tu, C.-Y., Zhou, C., Marsch, E., Wilhelm, K., Xia, L.-D., Zhao, L., Wang, J.-X.: 2005c, In: Lacoste, H. (ed.) Connecting Sun and Heliosphere, Proc. Solar Wind 11 / SOHO 16 SP-592, ESA, Noordwijk, 131.

Whang, Y.-C., Chang, C.-C.: 1965, J. Geophys. Res. 70, 4175.

Wilhelm, K., Marsch, E., Dwivedi, B.N., Hassler, D.M., Lemaire, P., Gabriel, A.H., Huber, M.C.E.: 1998, Astrophys. J. 500, 1023.

Wilhelm, K., Dammasch, I.E., Marsch, E., Hassler, D.M.: 2000, Astron. Astrophys. 353, 749.

Xia, L.-D., Marsch, E., Curdt, W.: 2003, Astron. Astrophys. 399, L5.

Zhang, J., Ma, J., Wang, H.-M.: 2006, Astrophys. J. 649, 464.

Zhang, J., Lin, G.-H., Wang, J.-X., Wang, H.-M., Zirin, H.: 1998a, Solar. Phys. 178, 245.

Zhang, J., Lin, G.-H., Wang, J.-X., Wang, H.-M., Zirin, H.: 1998b, Astron. Astrophys. 338, 322. 